\title{
PELIGRO Y RIESGO ESPECÍFICO ASOCIADO AL VOLCÁN CHAITÉN: PERSPECTIVA GEOLÓGICA DE LA VULNERABILIDAD EN EL ENTORNO DE UN VOLCÁN ACTIVO
}

\author{
LUIS E. LARA ${ }^{\mathrm{a}, \mathrm{b}}$ \& RODRIGO CALDERÓNa
}

\begin{abstract}
RESUMEN
La percepción del riesgo frente a los procesos naturales depende de múltiples factores cuya naturaleza es también variada. Un aspecto central en la evaluación del impacto potencial asociado a estos fenómenos descansa en la determinación objetiva de la amenaza misma, esto es, de la expresión concreta del proceso causante sobre el territorio. Una forma replicable de evaluación de la amenaza natural es el concepto de riesgo específico, que corresponde a la combinación del peligro y la exposición o vulnerabilidad física del territorio adyacente. Mientras el primero se refiere a la extensión del proceso mismo, la segunda remite a aquello potencialmente afectado antes de la aplicación de medidas de mitigación. En esa perspectiva, resulta de interés revisar la condición de riesgo específico representada por el volcán Chaitén y la situación en que se encuentra el pueblo homónimo y su población. Los antecedentes geológicos disponibles, particularmente aquellos obtenidos a partir de la erupción de año 2008, sugieren que el volcán Chaitén es uno de los 10 volcanes más activos del territorio, con un inédito historial de ciclos explosivos y reiterado impacto en los valles aledaños a causa de flujos detríticos e inundaciones. En un esquema estructurado de evaluación de riesgo específico, este centro resulta uno de los más significativos a nivel nacional en términos de frecuencia eruptiva, magnitud de los eventos documentados y variedad de los escenarios de peligro asociados. Asimismo, la vulnerabilidad física del entorno es comparativamente alta dada la localización del pueblo de Chaitén (ca. 5000 habitantes antes de la erupción) y su infraestructura asociada. En efecto, esta localidad representa un caso excepcional de cercanía extrema a un volcán activo en Chile y emula, a menor escala, la vulnerabilidad observada en localidades insertas en otras regiones del mundo como América Central o el sudeste asiático, cuya alta densidad de población explicaría en gran medida el efecto devastador de algunas erupciones recientes. Los datos geológicos ofrecen una perspectiva de largo plazo respecto de la amenaza natural que describe un escenario complejo y éste parece contrastar con el discurso frente al riesgo que sostienen los habitantes y algunas autoridades en esta localidad construida recién en la primera mitad del siglo XX. En consecuencia, una adecuada planificación del uso del territorio debería considerar los antecedentes geológicos e integrarlos con otros aspectos que finalmente permitan mitigar el impacto de futuros eventos eruptivos.
\end{abstract}

PALABRAS CLAVE: Peligro volcánico, Riesgo Específico, Vulnerabilidad.

a Programa de Riesgo Volcánico, SERNAGEOMIN.

b FONDAP-Centro Nacional de Investigación para la Gestión Integrada de Desastres Naturales. 


\section{HAZARDS AND SPECIFIC RISK RELATED TO CHAITEN VOLCANO: GEOLOGICAL PERSPECTIVE OF VULNERABILITY NEARBY AN ACTIVE VOLCANO}

\section{ABSTRACT}

Risk perception facing natural hazards depends on multiple factors which nature is also diverse. A key component of the impact assessment is the objective delineation of the hazards, meaning a clear definition of the hazardous processes and their spatial expression. A reproducible way for representing natural threat is the concept of specific risk, which is the product of hazards and exposure (or physical vulnerability) of the territory nearby. The first is dealing with causative process; the latter describe those things potentially affected before mitigation strategies. From this point of view, it is interesting to look at the specific risk posed by Chaitén Volcano and the situation of Chaitén village and its population. Geological evidence, specially that obtained after the 2008 eruption, suggest that Chaiten Volcano is one of the top ten more active volcanoes in Chile, with an exceptional record of explosive events and repeated impact on valleys because of the debris flows and floods. In a structured scheme of risk specific evaluation, Chaitén Volcano looks as one of the most prominent at national scale in terms of eruptive frequency, magnitude of the eruptions and variety of the hazards scenarios. Moreover, physical vulnerability of this village is high because of the location, population (ca. 5000 inhabitants before the last eruption) and infrastructure. In fact, this town is a rare case in terms of short distance to an active volcano and mimics the high exposure of some cities in other regions as Central America or South East Asia, where the high population density explains the deep impact of volcanic eruptions. Geological data provides a long-term perspective of the natural threat, which is high and contrasting with the risk perception of the inhabitants as deduced by their discourse and that of some authorities in this village, which was built just in the first half of the $20^{\text {th }}$ century.

Therefore, an appropriate land planning strategy should take into account geological evidence and to integrate that with other factors that finally promote a real mitigation of future eruptive events.

KEY WORDS: Volcano hazards, Specific risk, Exposure.

\section{INTRODUCCIÓN}

A nivel global, Chile es uno de los cinco países con más volcanes activos en el territorio nacional. En efecto, utilizando la definición técnica de Ewert (2007), existirian 91 volcanes activos en Chile (modificado de Lara et al. 2011). Sin embargo, en términos comparativos, la condición de riesgo real es modulada por la baja densidad de población en Chile, significativamente menor que aquella observada en los países del sudeste asiático o Centroamérica. Más aún, en Chile esta densidad disminuye con la reducción de la distancia a los centros volcánicos (Small \& Naumann, 2002), una condición opuesta a la observada en las regiones anteriores y que explicaría en buena medida los severos impactos que han causado históricamente las erupciones volcánicas en esos lugares. En efecto, erupciones como las del volcán Pinatubo en Filipinas, 1991 (e.g., Pallister et al. 1992) o aquella del Nevado del Ruiz en Colombia en 1983 (e.g., Voight, 1990) son ejemplo de la devastación causada en áreas densamente pobladas al pie de volcanes activos.

Una forma objetiva y replicable de evaluación de la amenaza natural es provista por el concepto de riesgo específico (UNDRO, 1979), que corresponde a la combinación ponderada del peligro y la exposición o vulnerabilidad física del territorio adyacente. El primero se refiere al proceso mismo y su expresión física en el territorio; la segunda remite a aquello potencialmente afectado antes de la aplicación de medidas de mitigación. En el contexto específico del riesgo volcánico, el riesgo específico o amenaza (en inglés threat) representada por un volcán individual corresponde a una categoría definida formalmente sobre la base de una colección de parámetros que evalúan objetivamente el peligro (en inglés hazard; basado en frecuencia eruptiva, magnitud máxima de las 


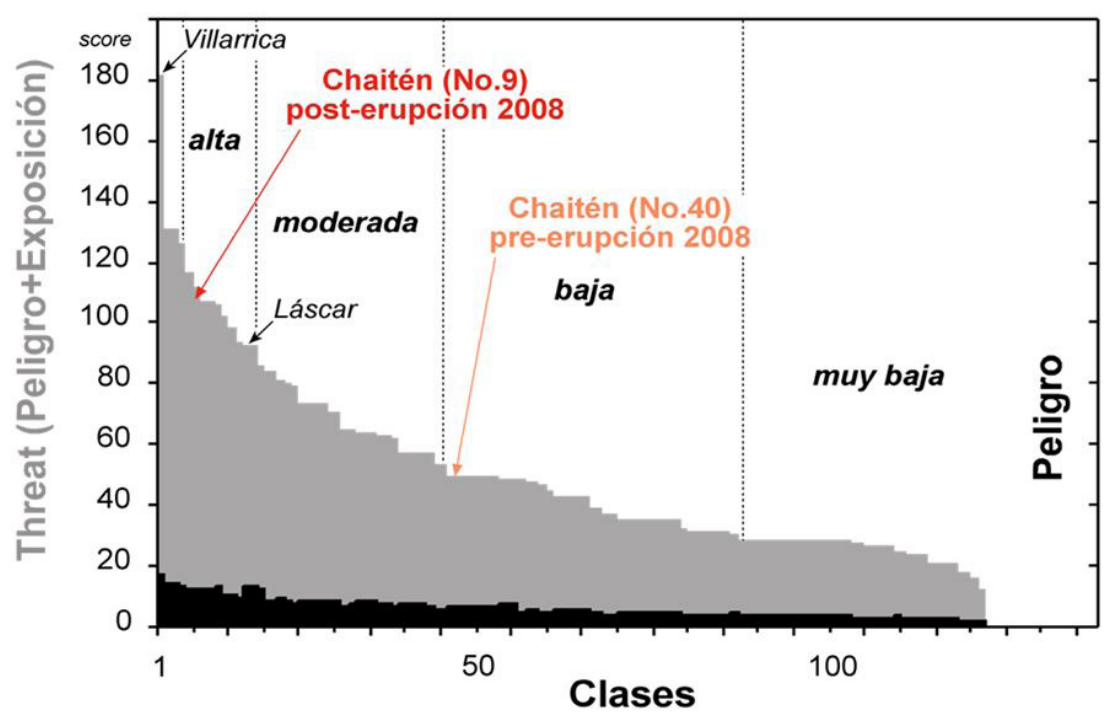

Fig. 1. Histograma de valores de Riesgo Específico y Peligro Volcánico (en gris y negro respectivamente; ver texto para detalles), modificado de Lara et al. (2011). El volcán Chaitén destaca entre los 10 volcanes más activos de Chile; una clasificación determinada fundamentalmente por recientes hallazgos geológicos que describen una alta frecuencia eruptiva con eventos de gran magnitud en los últimos 10 mil años y algunos similares a la erupción de 2008 ocurridos en el siglo XVII antes del

poblamiento moderno. Nótese la mayor dispersión de valores exhibida por el Riesgo Específico (gris), que permite efectivamente una distribución del puntaje y la subsecuente construcción del ranking, en contraste con los valores del Peligro Volcánico (negro) que resultan más uniformes. De lo anterior se desprende que los factores de exposición o vulnerabilidad controlan mayoritariamente el puntaje de Riesgo Específico y por lo tanto el análisis de la vulnerabilidad física aparece como de primer orden en una evaluación objetiva de la amenaza.

erupciones, presencia de glaciares en la cumbre, entre otros) y la exposición o vulnerabilidad física (densidad de población, presencia de infraestructura, entre otros) según la metodología propuesta por Ewert et al. (2005) y Ewert (2007) y para Estados Unidos y adaptada por Lara et al. (2011) al caso chileno.

En esa perspectiva, resulta de interés revisar la condición de riesgo específico representada por el volcán Chaitén y la situación en que se encuentra el pueblo homónimo y su población. En efecto, el volcán Chaitén está entre los más complejos en términos de riesgo específico al ubicarse en el quinto lugar del catálogo (Fig. 1) y genera en su entorno una condición de riesgo cuyas implicancias pueden observarse en diferentes ámbitos. En efecto, el puntaje generado por la evaluación objetiva de los diferentes parámetros que califican el peligro y exposición permite construir un ranking como el ilustrado en la figura 1 (actualizada de Lara et al. 2011 con información disponible al año 2013). En el mismo gráfico puede observarse que el peligro (en negro) presenta una tendencia similar a la del riesgo específico (en gris) pero las diferencias entre cada componente son significativamente más sutiles. En otras palabras, el factor que efectivamente diferencia a los volcanes y explica la distribución de los valores de riesgo específico es la exposición o vulnerabilidad. En este trabajo se analiza esa condición de riesgo específico desde la perspectiva del territorio próximo a un volcán activo, y particularmente de la condición de vulnerabilidad impuesta sobre el poblado de Chaitén por el volcán homónimo, exacerbada por la ocupación humana de un área de alto peligro volcánico.

\section{LA ERUPCIÓN 2008-2009 Y LA HISTORIA ERUPTIVA PREVIA}

El volcán Chaitén entró en erupción inesperadamente el 2 de mayo de 2008 generando una de las mayores erupciones de los últimos 30 años a nivel global. La erupción consistió en una fase explosiva con emisión de ceniza que duró alrededor de 2 semanas y generó un depósito de ca. $1 \mathrm{~km}^{3}$ de material piroclástico. La removilización de este material producto de las 


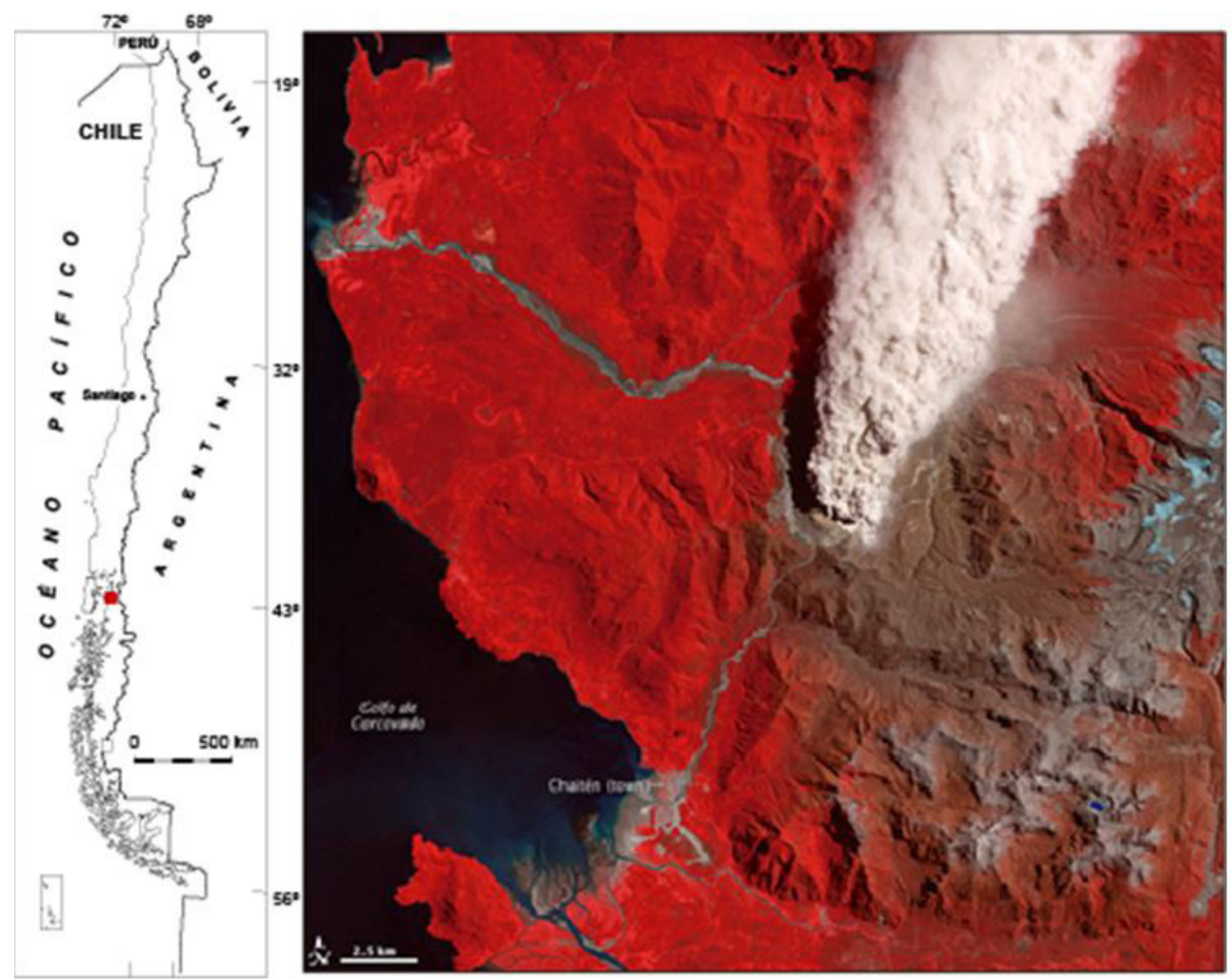

Fig. 2. Imagen ASTER en falso color adquirida el 19 de enero de 2009, más de 8 meses después de iniciada la erupción (cortesía de NASA/GSFC/METI/ERSDAC/JAROS). En ella puede observarse la aún activa, aunque baja, columna eruptiva y parte del domo construido en los primeros meses. Asimismo, se observa en tonos claros la cubierta de ceniza particularmente notoria hacia el oriente y las áreas afectadas por la removilización de cenizas en los valles principales, especialmente hacia el sur donde los sedimentos han construido un delta progradante.

intensas precipitaciones causó aluviones volcánicos $e$ inundaciones en los valles adyacentes dañando severamente la ciudad de Chaitén hacia el 12 de mayo, para entonces ya evacuada. Esta fase fue seguida por una fase efusiva de aproximadamente de casi 2 años de duración en la cual se formó un domo riolítico de ca. $0,8 \mathrm{~km}^{3}$, muy similar al existente antes del inicio de la erupción y construido hace alrededor de 5 mil años. Esta segunda etapa fue acompañada también de una columna eruptiva de menor altura que dispersaba intermitentemente ceniza (Fig. 2). El conocimiento previo sobre la historia eruptiva del volcán Chaitén era precario antes de la última erupción y la noción existente a la fecha sugería que la última actividad eruptiva relevante había ocurrido hace ca. 9 mil años (Naranjo \& Stern, 2004), sin indicios de actividad alguna en los últimos 5 mil años que corresponde al fechado de sitios arqueológicos cercanos con material lítico de obsidiana proveniente de este volcán (Stern et al. 2002). Más tarde, estudios de detalle han confirmado la ocurrencia de al menos 4 eventos relevantes en el Holoceno, dos de ellos correspondientes a erupciones plinianas (Newhall \& Self, 1982) ocurridas entre ca. 9,9 y 4,9 ka antes del presente y que generaron, además, corrientes piroclásticas en los valles adyacentes (Amigo et al. 2013; Watt et al. 2013). Particularmente interesante es un evento previo a la erupción de 2008 y de similar magnitud que habría ocurrido 
unos tres siglos antes del presente (Lara et al. 2013). En este último evento se habrían generado efectos similares a los observados en la erupción del año 2008-2009, incluida la generación de lahares o aluviones volcánicos en un proceso de agradación sucesiva que excedió el cauce activo del río Blanco y cubrió gran parte de la localidad (Pierson et al. 2013).

Por otra parte, un rasgo sobresaliente del volcán Chaitén es su tendencia a evacuar magmas de composición riolítica $\left(>70 \% \quad \mathrm{SiO}_{2}\right)$, que normalmente se asocian con erupciones inicialmente explosivas de mayor impacto potencial por su área de cobertura y especialmente por los riesgos implícitos para la salud de las personas debido al reducido tamaño de las partículas (Reich et al. 2009; Horwell et al. 2010). Y aunque la localidad de Chaitén y otros asentamientos menores se ubican a barlovento del centro de emisión, la proximidad a él y la morfología de la cuenca determinan que la redistribución del material volcánico a lo largo de los cauces pueda generar efectos tanto o más devastadores que la caída piroclástica primaria.

\section{EL POBLADO DE CHAITÉN Y SU CONDICIÓN DE RIESGO}

Hacia el año 2008, antes del inicio de la erupción, la localidad de Chaitén tenía alrededor de
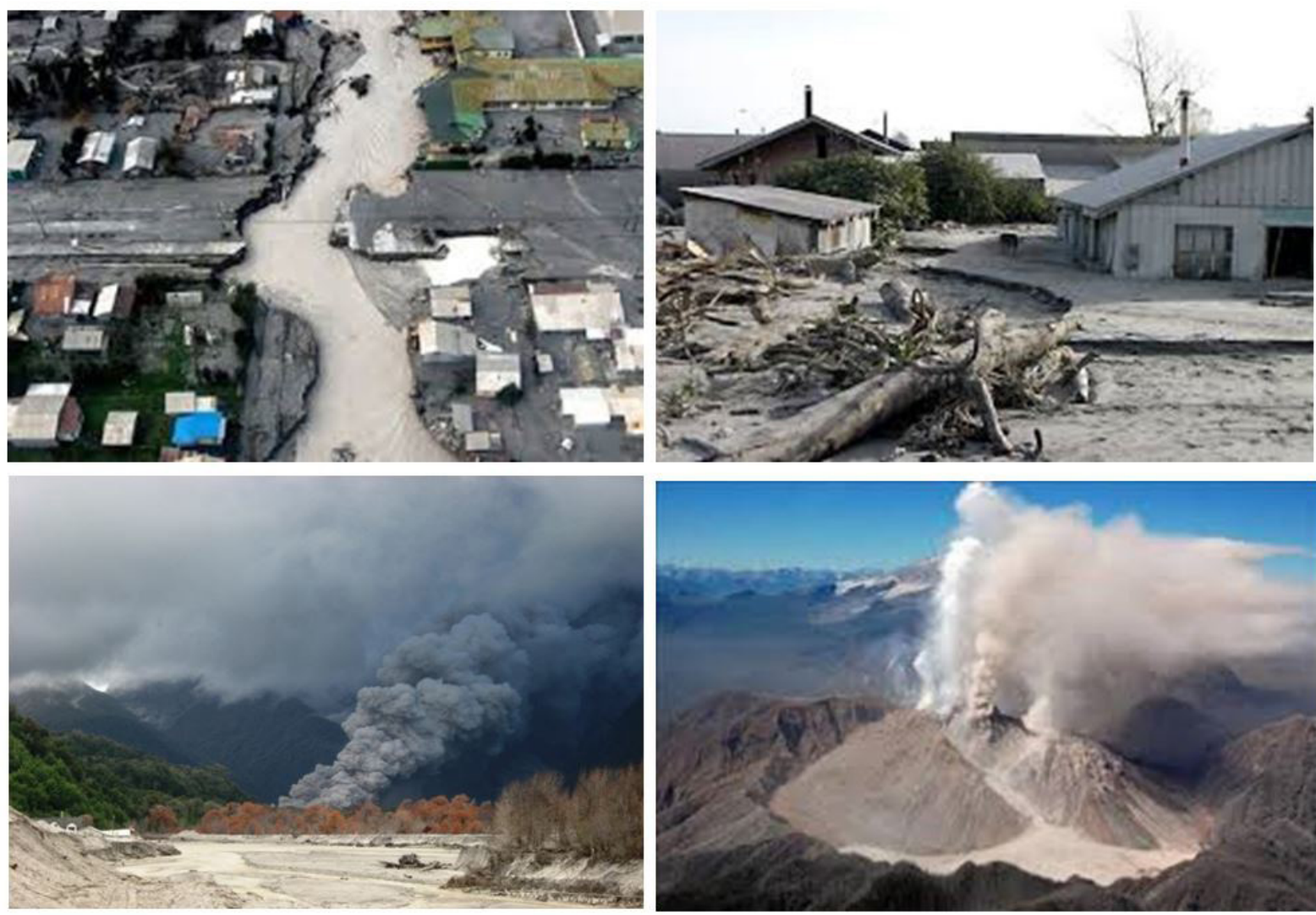

Fig. 3. Fotografías que ilustran el daño sufrido por la localidad de Chaitén durante la erupción 2008-2009. (arriba, izquierda) daño en el sector norte de la ciudad, donde un cauce secundario y más directo hacia el mar se estableció temporalmente (www.elrepuertero.cl). Existe evidencia geológica (Lara et al. 2013) de que este sector constituía un curso fluvial previo al emplazamiento de la población. (arriba, derecha) daño en el sector sur de la localidad, que corresponde al área de mayor acumulación de sedimentos en la planicie aluvial del río Blanco (www.taringa.net). (abajo, izquierda) valle del río Blanco con sedimentos acumulados por removilización del material piroclástico en la cuenca. En segundo plano se observa corriente piroclástica generada por colapso del domo nueve meses después del inicio de la erupción (gentileza de Dagoberto Guzmán). (abajo, derecha) vista panorámica del domo construido durante la erupción y los depósitos proximales de su colapso parcial, que constituyen una fuente secundaria de material piroclástico aportado a la cuenca. 


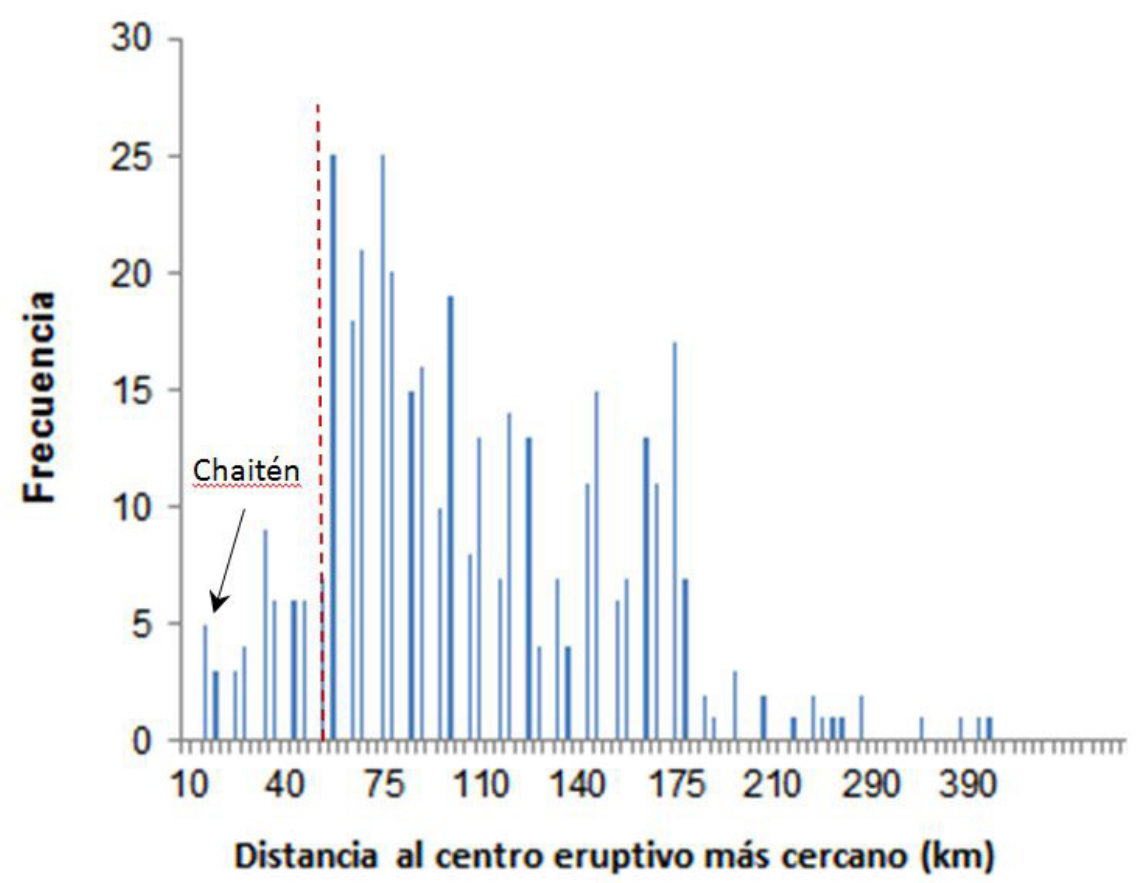

Fig. 4. Histograma de distancia y población para centros poblados de más de 5 mil habitantes (datos correspondientes al censo 200 en INE, 2003). Nótese la evidente concentración entre ca. 50 y $170 \mathrm{~km}$ de distacia a un volcán activo, lo que implica que gran parte de la población urbana de Chile se encuentra fuera de las áreas de peligro directo. Sin embrago, algunas localidades se encuentran en el radio crítico de $50 \mathrm{~km}$ (línea roja segmentada), entre ellas la localidad de Chaitén que corresponde a una de las más próximas.

5 mil habitantes. Fundada en 1940, su población comenzó a establecerse recién a principios del siglo XX y hacia 1946, con el inicio de la construcción del camino que uniría la costa con el interior, ella alcanzaba solo a un centenar de personas. Su población comenzó a consolidarse en los años 80 con la habilitación de la carretera austral y el servicio de transbordadores y alcanzó alrededor de 7 mil personas en la comuna hacia el año 2000, 4.625 de las cuales habitaban la zona urbana dañada posteriormente durante la erupción (INE, 2003). El daño sufrido por la ciudad fue severo, particularmente en su parte sur (Fig. 3), que aún muestra los efectos de la acumulación de material piroclástico movilizado por el río, cuyo cauce también fue modificado. La sección norte del poblado también fue afectada, pero en este lugar los daños han sido reparados y las autoridades consideran su rehabilitación completa. La apreciación anterior se contrapone con las evaluaciones de peligro y exposición realizadas en los años posteriores a la erupción, que determinaron incluso la proposición de un nuevo emplazamiento urbano en la localidad de Santa Bárbara, $25 \mathrm{~km}$ al norte y en un sector de menor peligro (www.plataformaurbana.cl).

En un contexto más general, y a diferencia del sudeste asiático o Centro américa, la población en Chile se sitúa mayoritariamente lejos de las áreas de influencia volcánica. En efecto, gran parte de las ciudades de más de 5.000 habitantes se sitúan en el valle central a ca. $80 \mathrm{~km}$ del arco volcánico, o en la costa a más de $120 \mathrm{~km}$ de él (Fig. 4). En términos relativos, Chaitén es una de las pocas ciudades de esa categoría que se ubica en el área de peligro proximal a solo $12 \mathrm{~km}$ de la zona apical del Chaitén. Asimismo, destaca también en la categoría de más de más de 1000 habitantes compartiendo ubicación con poblados menores instalados en las inmediaciones de algunos volcanes. Lo anterior supone una condición de riesgo severo por cuanto, estadísticamente, la zona proximal contenida en un radio de $30 \mathrm{~km}$ a un centro eruptivo concentra los mayores efectos directos durante eventos eruptivos de diferente magnitud (Blong, 1984), los que difícilmente se propagan más allá de $50 \mathrm{~km}$ en erupciones mayor magnitud. 
PELIGRO Y RIESGO ESPECÍFICO ASOCIADO AL VOLCÁN CHAITÉN

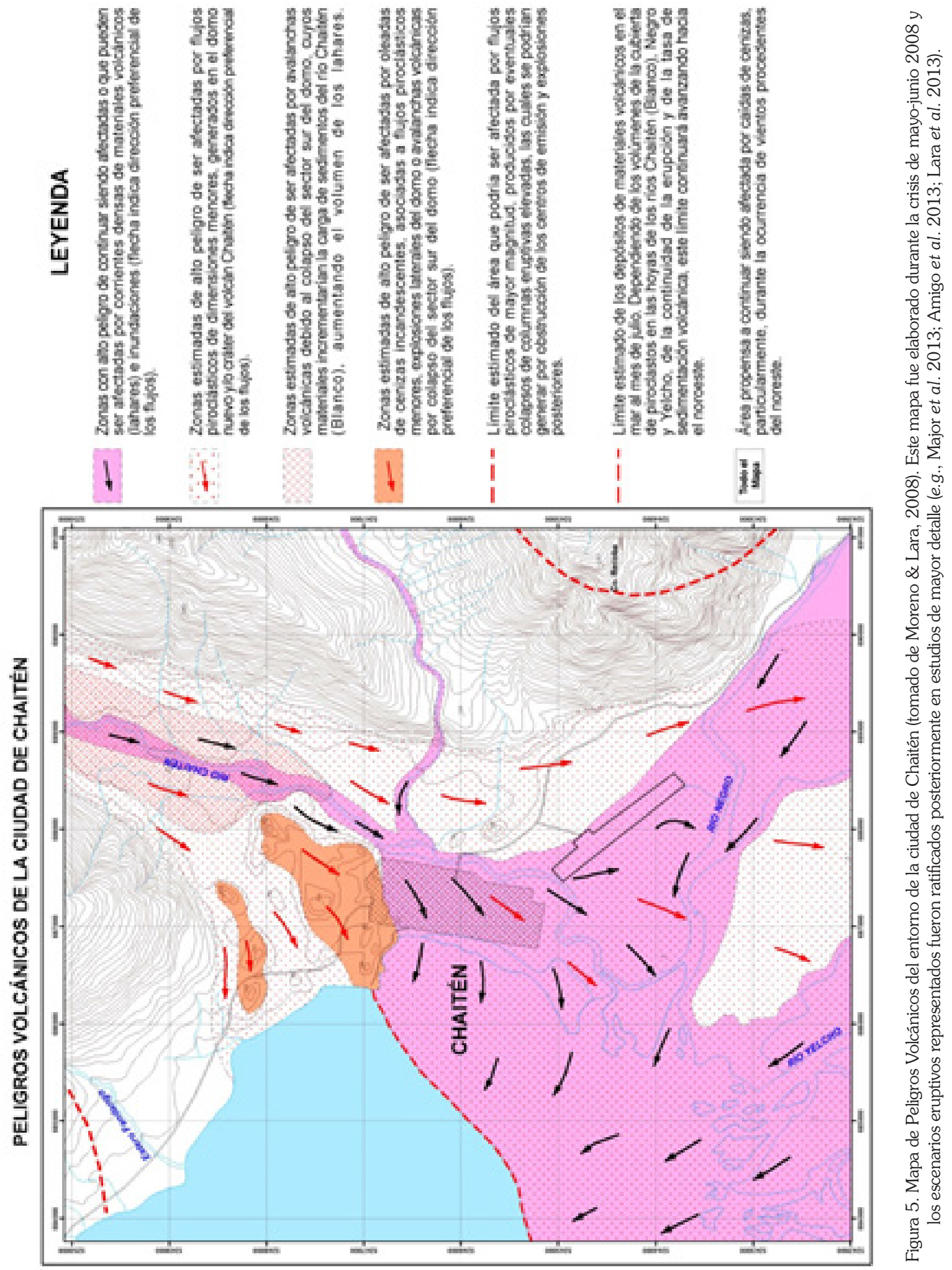


Y en efecto, el área inmediata al volcán Chaitén, incluida la localidad del mismo nombre, constituye un área de alto peligro frente a diferentes procesos compatibles con escenarios eruptivos moderados a severos como se deducen del registro geológico. La cartografía de peligros elaborada durante la crisis ilustra esa situación (Moreno \& Lara, 2008) indicando que toda la planicie aluvial está expuesta tanto al efecto de lahares como de flujos y oleadas piroclásticas.

\section{DISCUSIÓN: PERSPECTIVAS MULTIDISCIPLINARIAS}

Desde el siglo XVI, con el inicio del registro histórico introducido por misioneros y exploradores, existe alguna documentación de más de 400 eventos volcánicos (erupciones sensu stricto y otros eventos como explosiones menores o reactivaciones del sistema hidrotermal) observados en 39 centros volcánicos (Petit-Breuilh, 2004), la mayor parte de ellos ubicados en Chile central y centro-sur. De lo anterior se despende una relación magnitud-frecuencia que sugiere al menos una erupción de envergadura cada 8-10 años en el territorio nacional. No obstante esa alta frecuencia eruptiva, el registro global muestra cifras menores en términos de decesos o daños graves (Whitman, 2005), fundamentalmente atribuibles a la baja densidad de población en Chile. Por otra parte, los daños materiales asociados a erupciones volcánicas en Chile no han sido sistemáticamente evaluados. Al menos sobre la base de los casos más recientes (Hudson, 1991; Chaitén, 2008; Cordón Caulle, 2011), puede establecerse que el impacto sobre la agricultura, ganadería, turismo, energía y otros sectores productivos es importante y creciente en el tiempo por cuanto el desarrollo aumenta la exposición $\mathrm{y}$, consecuentemente, el riesgo (e.g., Chester et al. 2000).

Posiblemente la baja densidad de población en las áreas susceptibles de ser afectadas por erupciones volcánicas, sumada a la reducida experiencia que cada generación acumula en relación con crisis de este tipo, explique la alta tolerancia al riesgo que supone el poblamiento de zonas de alto peligro volcánico. Complementariamente, la falta de información apropiada y oportuna, la necesidad de explotación de los recursos naturales y la ausencia de un marco legal que vincule formalmente las condiciones de riesgo con la planificación territorial, entre otros factores, jugarían un rol importante en la percepción del riesgo que tienen comunidades como aquella que reside en la localidad de Chaitén.

\section{CONCLUSIONES}

La localidad de Chaitén se encuentra en una zona de alto peligro volcánico, determinada mediante estudios geológicos que han documentado una amplia sucesión de eventos eruptivos en el pasado. Particularmente compleja es la situación del poblado frente a la ocurrencia de lahares o aluviones volcánicos e inundaciones que suelen afectar primordialmente el cauce del río Blanco en cuya desembocadura se encuentra la zona urbana. En términos relativos, Chaitén es una de las localidades más expuestas a nivel nacional por encontrarse a solo $12 \mathrm{~km}$ de un volcán activo que, dada su configuración geológica, es propenso a la generación de erupciones explosivas. El nivel de riesgo específico asociado a este volcán es consecuentemente alto y tal condición debería considerarse en la planificación territorial.

\section{AGRADECIMIENTOS}

Parte de los antecedentes utilizados derivan del trabajo sistemático realizado en el marco del Programa de Riesgo Volcánico, parte de la Red Nacional de Vigilancia Volcánica que opera SERNAGEOMIN desde el año 2009. El análisis desplegado en este trabajo forma parte de una aproximación multidisciplinaria impulsada por CIGIDEN (www.cigiden.cl) y consecuentemente agradecemos al Programa FONDAP de CONICYT por el apoyo financiero de nuestra investigación a través del proyecto FONDAP 15110017. Se agradece a Adriana Muñoz como editora especial.

\section{BIBLIOGRAFÍA}

\footnotetext{
Amigo, Á., Lara, L. E., \& Smith, V. C. (2013). New Holocen record of large eruptions from Chaitén and Michinmahuida volcanoes, Chile. Andean Geology, 40(2), $227-$ 248.

Blong, R. J. (1984). Volcanic Hazards. A Sourcebook on the Effects of Eruptions. Sidney: Academic Press.
} 
Chester, D. K., Degg, M., Duncan, A. M., \& Guest, J. E. (2000). The increasing exposure of cities to the effects of volcanic eruptions: a global survey. Envirnmental Hazards, 2, 89-103.

Ewert, J. W. (2007). System for Ranking Relative Threats of US Volcanoes. Natural Hazards Review, 8, 112-124.

Ewert, J. W., Guffanti, M., \& Murray, T. L. (2005). NVEWS: An Assessment of Volcanic Threat and Monitoring Capabilities in the United States: Framework for a National Volcano Early Warning System. U.S. Geological Survey, Open File Report 2005-1164.

Horwell, C. J., Le Blond, J. S., Michnowicz, S. A., \& Cressey, G. (2010). Cristobalite in a rhyolitic lava dome: evolution of ash hazard. Bulletin of Volcanology, 72(2), 249-253.

Instituto Nacional de Estadísticas. (2003). Mapa interactivo Censo 2002. Obtenido de http://www.ine.cl/canales/chile_estadistico/censos_poblacion_vivienda/censo2002/mapa_interactivo/mapa_interactivo.htm

Lara, L. E., Moreno, R., Amigo, Á., Hoblitt, R. P., \& Pierson, T. C. (2013). Late Holocene history of Chaitén Volcano-new evidence for a 17 th-century eruption. Andean Geology, 40(2), 249-261.

Lara, L. E., Orozco, G., Amigo, Á., \& Silva, C. (2011). Peligros volcánicos de Chile. Servicio Nacional de Geología y Minería, Carta Geológica de Chile, Serie Geología Ambiental, No., p., 1 mapa escala 1:2.000.000. Santiago.

Naranjo, J. A., \& Stern, C. R. (2004). Holocene tephrochronology of the southernmost part $\left(42^{\circ} 30^{\prime}-45^{\circ} \mathrm{S}\right)$ of the Andean Southern Volcanic Zone. Revista Geológica de Chile, 31(2), 225-240.

Newhall, C., \& Self, S. (1982). The Volcanic Explosivity Index (VEI): An estimate of explosive magnitude for historical volcanism. Journal Geophysical Research, 87, 1231-
1238 .

Pallister, J. S., Hoblitt, R. P., \& Reyes, A. G. (1992). A basalt trigger for the 1991 eruptions of Pinatubo Volcano? Nature, 356 (6368), 426-428.

Petit-Breuilh, M. E. (2004). La historia eruptiva de los volcanes hispanoamericanos (Siglos XVI al XX). Cabildo Insular de Lanzarote-Casa de los volcanes: Servicio de Publicaciones del Exmo.

Pierson, T. C., Major, J. J., Amigo, Á., \& Moreno, H. (2013). Acute sedimentation response to rainfall following the explosive phase of the 2008-2009 eruption of Chaitén volcano, Chile. Bulletin of Volcanology, 75(5), 1-17.

Reich, M., Zúñiga, A., Amigo, Á., Vargas, G., Morata, D., Palacios, C.,... Garreaud, R. D. (2009). Formation of cristobalite nanofibers during explosive volcanic eruptions. Geology, 37(5), 435-438.

Small, C., \& Naumann, T. (2001). The global distribution of human population and recent volcanism. Environmental Hazards, 3, 93-109.

Stern, C., Narrova, X., \& Muñoz, J. (2002). Obsidiana gris translúcida del volcán Chaitén en los sitios arqueológicos de Quilo (Isla Grande de Chiloé) y Chanchán (X Región), Chile, y obsidiana del Mioceno en Chiloé. Anales del Instituto de la Patagonia, 30, 167-174.

UNDRO. (1979). Disaster Prevention and Mitigation: A Compendium of Current Knowledge 7 (Aspectos Económicos). Nueva York.

Voight, B. (1990). The 1985 Nevado del Ruiz volcano catastrophe: anatomy and retrospection. Journal of Volcanology and Geothermal Research 42(1), 151-188.

Watt, S., Pyle, D., \& Mather, T. (2013). Evidence of mid- to late-Holocene explosive rhyolitic eruptions from Chaitén volcano, Chile. Andean Geology, 40(2), 249261. 